\title{
Early Detection of Mild Cognitive Impairment (MCI) in Primary Care
}

\author{
M.N. Sabbagh ${ }^{1}$, M. Boada 2 , S. Borson ${ }^{3}$, M. Chilukuri 4 , B. Dubois 5 , J. Ingram ${ }^{6}$, A. Iwata 7 , A.P. Porsteinsson ${ }^{8}$, \\ K.L. Possin ${ }^{9}$, G.D. Rabinovici ${ }^{9}$, B. Vellas ${ }^{10}$, S. Chao ${ }^{11}$, A. Vergallo ${ }^{12}$, H. Hampel He $^{12}$
}

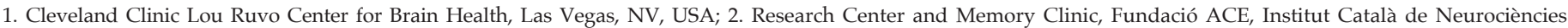

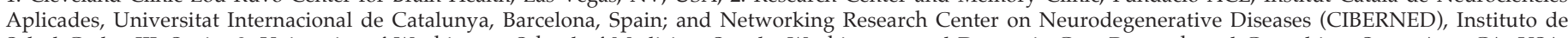

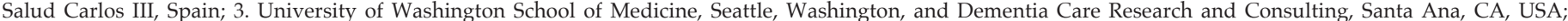

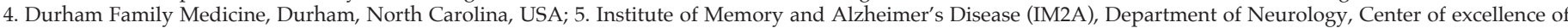

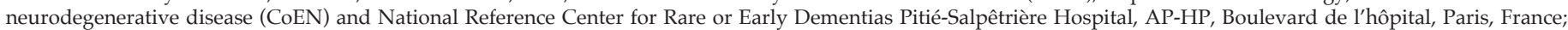

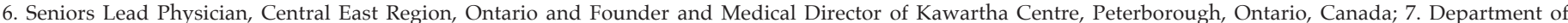

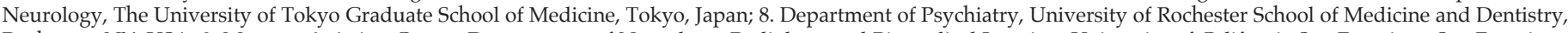

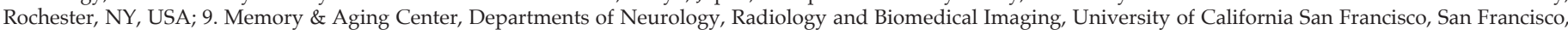

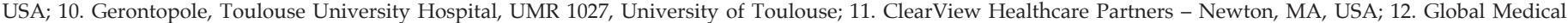
Affairs, Neurology Business Group, Eisai Inc., Woodcliff Lake, New Jersey, USA

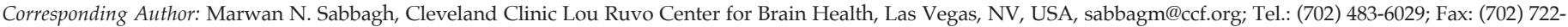
6584

J Prev Alz Dis 2020;3(7):165-170

Published online April 6, 2020, http:/ / dx.doi.org/10.14283/jpad.2020.21

\begin{abstract}
Mild cognitive impairment (MCI) is significantly misdiagnosed in the primary care setting due to multi-dimensional frictions and barriers associated with evaluating individuals' cognitive performance. To move toward large-scale cognitive screening, a global panel of clinicians and cognitive neuroscientists convened to elaborate on current challenges that hamper widespread cognitive performance assessment. This report summarizes a conceptual framework and provides guidance to clinical researchers and test developers and suppliers to inform ongoing refinement of cognitive evaluation. This perspective builds upon a previous article in this series, which outlined the rationale for and potentially against efforts to promote widespread detection of MCI. This working group acknowledges that cognitive screening by default is not recommended and proposes large-scale evaluation of individuals with a concern or interest in their cognitive performance. Such a strategy can increase the likelihood to timely and effective identification and management of MCI. The rising global incidence of $\mathrm{AD}$ demands innovation that will help alleviate the burden to healthcare systems when coupled with the potentially near-term approval of disease-modifying therapies. Additionally, we argue that adequate infrastructure, equipment, and resources urgently should be integrated in the primary care setting to optimize the patient journey and accommodate widespread cognitive evaluation.
\end{abstract}

Key words: Alzheimer's disease, mild cognitive impairment, cognitive screening, disease-modifying.

\section{Introduction}

$\mathbf{M}$ ild Cognitive Impairment (MCI) is a syndrome defined by clinical, cognitive, and functional criteria and is characterized by an objective cognitive decline in one or more cognitive domains without any significant impairment in daily-life activities. MCI may be associated with a Received January 9, 2020 variety of underlying causes, including Alzheimer's pathophysiology $(1,2)$.

Late-stage clinical development of drugs with a disease-modifying effect represents unprecedented hope for individuals suffering from Alzheimer's disease $(\mathrm{AD})$, particularly at preclinical or prodromal stages (i.e., MCI due to AD [MCI-AD]). In addition, the expanding knowledge on non-pharmacological approaches to cognitive decline (e.g., lifestyle-oriented treatments, non-invasive brain stimulation) suggests the possibility to treat secondary causes of MCI. This report represents the second part of a three-part consensus perspective on testing for MCI and is focused on the primary care setting. The suggestions and opinions within these publications represent the consensus opinion of a working group comprised of international experts on MCI and AD that was convened in April 2019 to discuss the challenges of detecting MCI at a large-scale and the potential solutions to overcoming these barriers.

Recommendations described here focus on improvements to MCI detection that may be feasible and ready for widespread use in the near-term (i.e., within approximately three years). The implementation of a system of healthcare delivery focused on dementia screening and large-scale cognitive screening is necessary to accommodate the global rising incidence of $\mathrm{AD}$, and to prepare the public and healthcare providers for the availability of disease-modifying therapies for AD. Blood-based and biologic biomarkers are expected to play a key role in this paradigm shift. Indeed, bloodbased biomarker panels are widely accessible, minimally invasive, and less time- and cost-consuming than cerebral spinal fluid (CSF) and neuroimaging assessments. To that end, we have outlined current barriers to the timely and accurate detection of MCI and MCI-AD, provided potential solutions, identified methods and emerging technologies to improve cognitive evaluation, 
and estimated potential timelines for accomplishing an optimal care pathway for managing $\mathrm{MCI}$ and MCI-AD at a large-scale.

\section{Current landscape}

\section{Barriers Related to Physician Training and Support}

The expert panel identified a wide range of barriers, including expertise, schedule, and available assessment tools, that often prevent primary care physicians (PCPs) from evaluating cognition.. The short duration of most primary care visits (frequently less than twenty minutes) represents one of the key logistical barriers to the establishment of cognitive evaluation in a primary care setting. The high prevalence of comorbidities among older adult individuals intensifies this challenge. Cognitive pathways require access to collateral informants, usually family members. Physicians may lack sufficient access to these collateral sources who are close enough to the individual to provide accurate longitudinal insight into his or her cognitive performance and functional abilities. Given these logistical barriers, PCPs may not consider assessing an individuals' cognitive performance within the context of a standard appointment feasible.

Separate from logistical concerns, PCPs are also likely to encounter barriers around their comfort with cognitive assessment and/or motivation to assess cognition (3). Importantly, many PCPs have reported limited confidence in cognitive assessment. Training programs for Primary Care providers incorporate limited exposure to these skills. As a result, many PCPs are left feeling poorly equipped, inexperienced, or uncomfortable about monitoring cognitive performance $(4,5)$. In addition, if cognitive impairment is detected, PCPs may face uncertainty about what next steps to pursue (e.g., how to appropriately explain any test results, whether or not to refer to a specialist). Finally, PCPs likely face low motivation to evaluate an individual's cognitive status, given uncertainty around whether or not identifying MCI provides a clear benefit to the individual. Without effective treatments for MCI, detecting MCI may be perceived only to be detrimental to individuals and their family members. While emergence of a novel diseasemodifying therapy may ultimately address physician motivation, challenges to confidence and familiarity with cognitive assessment may require large-scale training efforts.

\section{Barriers Related to Healthcare Systems}

Barriers associated with healthcare systems also significantly limit widespread early detection of MCI, as exploring all cognitive domains and quantifying overall cognitive performance is currently a timeconsuming process. In the context of an individual physician's office, current medical practice can limit the use of MCI in many different ways. Poor integration of cognitive assessments with EMR systems creates a significant administrative burden, as substantial clerical work is needed to document the output of a cognitive performance assessment. In addition, lack of proper integration with the EMR system also limits the ability to track an individual's cognition over time, which in turn limits the utility of cognitive evaluation. In some circumstances, testing tools are poorly designed and/or unintuitive for users.

This increases system burden due to cost (e.g. administration time, training, clerical burden etc.), which decreases the frequency of cognitive performance assessment. On the macro scale, inadequate reimbursement of costs associated with assessing cognitive function and providing post-diagnostic care, including physician time, significantly decreases the incentive for wide adoption of MCI detection. Consistent, reliable reimbursement of comprehensive assessment and cognitive testing by payers is therefore required to support extensive evaluation of cognition in the primary care setting.

\section{Barriers Related to Test Design and Validation}

The limited length of time of the average PCP visit requires tests to be conducted in 10 minutes or less. This constraint introduces a major limitation, as cognition is multifaceted, and many different cognitive domains can be impacted by MCI. Testing all domains of cognition in a short test is likely not feasible, so tools must strike a proper balance between time and depth of testing to maximize their utility.

Additionally, many cognitive tests have demonstrated limited value when deployed in a heterogeneous patient population. This limitation results from initial development and validation with highly homogeneous populations in mind - specifically, highly-educated English-speakers. Effective tests must be usable in a broader community that includes individuals across multiple levels of educational attainment, various races and ethnicities, and multiple languages (including varying familiarity with English). Validation in homogeneous populations can lead to the development and use of tools that are significantly less accurate than expected when used in a diverse patient population; for example, a patient with fewer years of education may score artificially low on a screening tool developed and validated in college graduates. Many tests also lack validation in multiple languages, which prevents standardization across communities and countries. 


\section{Barriers Related to MCI}

Early detection of MCI is also inherently challenging due to barriers associated with the disease itself. Symptoms related to the initial onset of MCI can vary significantly between individuals, depending on etiology, cognitive reserve, and variable demands of dayto-day living, among other factors. Additionally, MCI can be less relevant than other medical comorbidities, contributing to a different medical prioritization ahead of monitoring cognition. Furthermore, care partners and patients are likely to be particularly sensitive about cognitive performance in comparison to other health concerns. Patient concerns may result in a scenario where a physician is hesitant to discuss the cognitive performance assessment or the implications these have on other skills (such driving) with patients due to concern about compromising the physician-patient relationship. Similarly, individuals may actively avoid discussing cognitive performance with their physician due to concerns about the implications of cognitive assessment and/or perceived stigma associated with cognitive impairment. All of these issues can limit the utility of even clinically useful tests due to lack of use and compliance.

\section{Parameters of an ideal tool}

To help guide the refinement of existing cognitive performance evaluations or development of novel tools, we have outlined the parameters of an "ideal" MCI detection tool. This guidance is intended to offer potential solutions to the barriers currently facing MCI detection. While this working group does not recognize a single assessment that meets all of these criteria, multiple cognitive performance assessments include components of an "ideal" tool, suggesting that these promising tools may approach the "ideal" profile with minor refinement and / or additional validation.

\section{Test Methodology}

Similar to previous recommendations (6), this panel agreed that a tool for the detection of MCI would ideally incorporate three critical components: cognitive assessment, functional questionnaires, and clinical history-taking. First, cognitive assessment refers to directly assessing cognitive function through objectively evaluated tasks, such as a word recall task, clock-drawing task, etc. To meet the criteria for defining MCI versus dementia, a cognitive tool should also encompass multiple cognitive domains; our working group recommends that, at minimum, memory and executive function be assessed. Ideally, measures of visuospatial and language skills would also be included. Many currently-available cognitive tests encompass multiple cognitive domains, including Cognigram (offered by CogState) (7), CogniSense (offered by Quest Diagnostics) (8), and CANS-MCI (offered by Screen Inc.) (9). Emerging computer-based neuropsychological approaches may also be considered. The Toronto Cognitive Assessment (TorCA) is an example of a computer-based platform integrated across multiple sites, providing consistent analysis and interpretation (10).

Second, functional questionnaires refer to tools that ask the individual or a family member about activities of daily living, which by definition must be returned to diagnose MCI but must be impacted by cognitive challenges to permit the diagnosis of dementia (e.g., ability to carry out financial tasks, driving, shopping). A long-standing example is the Functional Activities Questionnaire (FAQ), a 10-question form with questions around ability to go shopping or prepare a meal (11).

Third, clinical history-taking aims to identify comorbidities and their impact on function and to understand whether the individual himself or a family member has noticed a change in cognitive function over time. Questionnaires such as the AD8 or IQCODE can be utilized to facilitate clinical history-taking (12-14).

Importantly, clinical history-taking will help identify individuals with MCI but may also help identify individuals with subjective cognitive decline (SCD), which is often a precursor to MCI and can be considered a preclinical phase of $\operatorname{AD}(15,16)$. An ideal cognitive assessment would encompass all three of these components; notably, a single questionnaire could incorporate both a functional component as well as questions for clinical history taking. An ideal tool would include a core assessment based on assessment of the individual him/herself, with an optional module that could incorporate feedback from a family member when possible. Furthermore, an ideal tool would allow the family member to complete a survey remotely (e.g., through an online form linked to the assessment). A tool that incorporates these components and features is likely to achieve compelling accuracy, even in individuals with subtle cognitive decline.

\section{Logistics}

The working group recommends several logistical characteristics that may optimize ease of use and minimize the time burden associated with detection of MCI. Tests should 1) be administered digitally on a laptop, tablet, or smartphone to facilitate widespread use and allow testing to scale, 2) require less than ten minutes, 3) not require a physician (i.e., should be selfadministered or conducted by a technician or nurse). Upon completion, the assessment should automatically create a report that outlines next steps in care specific to each healthcare system and/or region. The automated report should be integrated with EMR systems and should be available to the PCP instantly so that they 
can easily discuss the results with the individual at the beginning of the patient visit. The cost of administering a test also can be a significant logistical consideration. While this panel recognizes that a highly accurate, validated, and well-designed test will command a higher price than other options, an "ideal" assessment would be offered at a low price point and/or would be reimbursed by payers to maximize access and use of the assessment. Multiple currently available tools align with one or more of these criteria given the recent increase in creation of digitally administered tests. CogniSense, offered by Quest Diagnostics, meets the above mentioned criteria and can be automatically integrated into EMR (17). This panel recommends that creators of assessment tools seek to incorporate features that will optimize functionality and minimize administrative burden associated with detection of MCI.

\section{Validation}

An ideal tool would be validated in a diverse population (i.e., varied cultural and educational backgrounds) and validated (not merely translated) in multiple languages. Validating studies should be conducted in populations representative of the distribution of mild cognitive impairment, dementia, and normal cognition in a primary care setting, not in populations enriched for subjects with cognitive impairment. Recently, creators of the Brain Health Assessment (BHA; developed at UCSF) utilized this approach to validate the accuracy of the BHA in a clinically-representative patient population $(\sim 55 \%$ healthy controls, $\sim 30 \% \mathrm{MCI}$ individuals, $10 \%$ dementia patients, and $\sim 5 \%$ with subjective cognitive concerns) (18). If a tool is to be utilized broadly in a primary care setting, moderate specificity may be acceptable, given that additional downstream assessment will occur.

High sensitivity will ensure that a high proportion of suitable individuals receives follow-up assessment. The members of this working group acknowledge that validation of a cognitive performance assessment can be challenging. In the absence of an accepted "gold standard" test (or set of tests), multiple tests may be considered suitable. This working group recommends that novel tests continue to be validated in comparison to diagnosis based on a detailed clinical examination supported by multiple long-standing assessment tools.

\section{Optimal care pathway}

In light of these barriers to MCI detection, the expert panel agreed that it will be necessary to clarify the "optimal care pathway" for the detection of early cognitive impairment. While further work will be needed to understand how cognitive performance assessments can best be integrated across healthcare systems, this panel discussed select characteristics of an "ideal" care pathway. First, given the enduring uncertainty around whether universal screening is beneficial, this group recognized that the most valuable early detection pathway would begin with individuals who already have a cognitive performance concern (initiated either by the individual themselves, a family member, or the healthcare provider) or individuals who actively opt-in to cognitive assessment. Indeed, it is likely that individuals with a concern about their own cognitive performance are most likely to benefit from cognitive assessment given that subjective memory complaint (i.e., a self-reported loss of memory performance without objective cognitive decline) represents a condition at-risk for developing $\mathrm{MCI}$ in general. Moreover, SCD may underlie the beginning of the AD clinical continuum $(1,19,20)$. Therefore, largescale cognitive screening may also involve subjects with SCD in light of initiating therapeutic interventions targeting preclinical AD. The assessment of the SCD condition starts from a self-reported dysfunction and requires an assessment of the whole cognitive battery test employed for investigating if an objective cognitive decline exists (and per definition as to be negative). Therefore, the screening of potential preclinical stages of $\mathrm{AD}$ would be included in the same protocol to identify MCI.

Individuals identified with MCI then must be efficiently and thoroughly evaluated and guided toward appropriate next steps. We discussed two potential pathways: in one pathway, individuals undergo a brief cognitive assessment in parallel with a standard primary care appointment (e.g., a Medicare Annual Wellness Visit in the U.S. or MINT Clinic in Canada). In a second pathway, the individual may schedule a separate optional cognitive assessment appointment with their physician. In both pathways, the brief cognitive assessment should be either self-administered or administered by trained medical personnel (potentially a medical technician or nurse).

In some medical systems, creation of embedded nursing personnel trained to carry out cognitive evaluations on an as-needed basis have been well received. Creation of primary care clinicians with special training and expertise in this area, coupled with specific memory teams in primary care, has received wide-spread endorsement (https:/ / www.hqontario.ca/QualityImprovement/Quality-Improvement-in-Action/ARTIC/ ARTIC-Projects / Primary-Care-Collaborative-MemoryClinics). System-based changes in healthcare delivery are required to achieve the desired impact. Ultimately, individual choice of cognitive screening tools should decrease as pathways, including assessments, aligned with prior parameters become operationalized.

Importantly, an optimal care pathway must effectively support individuals and their caregivers following the cognitive assessment. After the assessment, the physician must allow sufficient time to help the individual 
understand the results and to provide guidance on next steps. Depending on the healthcare system and the capabilities of each primary care practice, this pathway may include further assessment in the primary care office, referral to a specialist, or simple monitoring of cognitively intact individuals to potentially detect a decline in subsequent years. To ensure maximal compliance with testing practices, we recommend that the next steps be clearly outlined by each healthcare system, in EMR systems, and/or by evaluative tools themselves.

\section{Potential Future Blood-based Testing}

If blood-based biomarkers become available earlier than anticipated, this is expected to significantly accelerate the diagnosis of AD and improve global accessibility of diagnostic tools. However, despite the promise of blood-based testing, our panel agreed that cognitive performance assessments will remain critical for distinguishing $\mathrm{MCI}$ and $\mathrm{MCI}-\mathrm{AD}$ in the future, even after blood-based biomarkers are implemented into primary care practice. Cognitive testing and functional evaluation will remain necessary to understand the individual's current cognitive performance, monitor changes in cognitive function, and identify cognitive changes not associated with a distinct biological signature (e.g., secondary causes of MCI).

Blood-based biomarkers are expected to facilitate critical clinical solutions catalyzed by the global threat of the evolving $\mathrm{AD}$ epidemic. The negative predictive value of blood-based biomarkers will support early screening and identification of individuals with a very low probability of developing AD-related pathophysiology and increase the probability that individuals with AD pathophysiology are being selected for further investigation by using more specific, expensive, and/ or more invasive methods with reduced accessibility (e.g., PET imaging or CSF assessment). Blood-based biomarkers have excellent potential to be routinely and rapidly assessed in all healthcare settings and in asymptomatic individuals due to minimal invasiveness, cost-efficiency, accessibility (i.e., blood can even be withdrawn in an individual's home), and reduced time and resource utilization compared with neuroimagingand CSF-based techniques used for AD.

Indeed, growing optimism exists regarding blood-based biomarkers reflecting distinctive AD pathophysiological mechanisms, supported by increasing evidence that core biomarkers and proteins associated with inflammatory and neurodegenerative pathways can be detected in blood $(21,22)$. While the sensitivity of conventional immunoassays may be insufficient to detect changes in the blood in individuals with $\mathrm{MCI}$ and $\mathrm{MCI}-\mathrm{AD}$, promising assays using novel technologies are in development. Multiplex digital ELISA platforms (e.g., Quanterix ${ }^{\circledR}$ Simoa $\left.{ }^{\circledR}\right)$ have been used by multiple groups to distinguish $\mathrm{MCI}$ and MCI-AD using blood levels of various proteins, including neurofilaments, $A \beta$, and tau (23-25). Another approach that has improved sensitivity of immunoassays is immunomagnetic reduction (IMR) via the use of superconducting quantum interference devices (SQUIDs). In one study, use of an IMR-SQUID assay combining analysis of $\mathrm{A} \beta 42$ and tau, enabled detection of AD with an AUC of 0.98 (26). Promising results also have been achieved in the academic setting using mass spectrometry. Multiple publications have distinguished between $\mathrm{AD}, \mathrm{MCI}$, and normal controls using ratios of $\mathrm{A} \beta$ and APP isoforms (27), or by using composite protein profiles detected via mass spectrometry (28). While further research is needed, development of an accurate, cost-effective, scalable blood-based biomarker for cognitive decline will shift the clinical paradigm dramatically, increasing diagnostic confidence and comfort of physicians, and integrating the novel biomarker test into the diagnostic paradigm will be critical.

\section{Discussion}

As outlined above and in the first article of this series, anticipated societal and medical changes (e.g., aging populations and potential advancements in management of $\mathrm{MCI}$, respectively) will necessitate a significant improvement in the early detection of MCI. As $\mathrm{PCP}^{\prime} \mathrm{s}$ are the initial point of contact, especially for stoutly progressive chronic illness with subtle initial presentations, the PCP is likely the central player in initial identification and management of MCI. Importantly, PCPs are often inadequately supported to allow widespread evaluation of cognitive and functional performance, and cognitive assessment tools themselves are not optimally designed to support widespread use in the primary care setting. While tools evolve, health care developments and spending should focus on improving training on identification and management of MCI at the PCP's office. Care pathways and staffing at the primary care physician's office to support PCP management of cognition are needed (e.g., ensuring visits are long enough to allow for a cognitive evaluation). A critical need exists to refine cognitive performance assessments and to validate tools in diverse, representative populations, ideally in multiple languages. Test makers should also be aware of the barriers that limit early detection of MCI, including barriers associated with the primary care setting and with broader healthcare systems, so that tests can be designed to counteract or limit these barriers

This working group recommends that key stakeholders representing PCPs, regulatory stakeholders, test makers, and patient advocates collectively take action to improve the use and quality of tests for the detection of MCI. In the next and final article of this series, we shall explore the role and value of direct-to-consumer cognitive testing options. 
Acknowledgements and funding: Medical writing support, under the direction of the authors, was provided by ClearView Healthcare Partners, LLC, funded by Eisai Inc., in accordance with Good Publication Practice (GPP3) guidelines.

Disclosures: $\mathrm{MB}$ is affiliated with the Research Center and Memory Clinic Fundació ACE, Institut Català de Neurociències Aplicades, Universita Internacional de Catalunya, Barcelona, Spain; and with the Networking Research Center on Neurodegenerative Diseases (CIBERNED), Instituto de Salud Carlos III, Spain. Private research funding sources include Grifols SA; Caixabank S.A.; Life Molecular Imaging; Araclon Biotech; Laboratorios Echevarne; Festival Castell Paralada; Bonpreu/Esclat; and Famila Carbó. Public grants include those from Instituto de Salud Carlos III. Ministerio de Salud. Gobierno de España; Dirección General de Farmacia. Ministerio de Salud. Gobierno de España; and European Commission:H2020 program, Innovative Medicine Initiative (IMI-2), and ERA-NET NEURON program, European Marie Sklodowska Curie. Advisory work includes that for Araclon Biotech, Biogen, Bioibérica, Eisai, Grifols, Lilly, Merck, Nutricia, Roche, Oryzon, Schwabe Farma, Servier, and Kyowa Kirin BD is affiliated with the Institute of Memory and Alzheimer's Disease (IM2A), Department of Neurology, Salpêtrière Hospital, AP-HP, Sorbonne-Université, Paris, France. JI is affiliated as an Assistant Professor-Adjunct (Group1) with the Department of Family Practice at Queen's University. She was also a panelist for Hoffman-La Roche Limited, Ottawa, on an Alzheimer's Disease Panel. She is involved with the Canadian Consortium on Neurodegeneration in Aging (CCNA), Extension Study as a Co- Investigator and Research Coordinator. AI received lecture fees from Eisai, Janssen, Otsuka, Eli Lilly, MSD, ChugaiRoche, Daiichi-Sankyo, Alnylam, Takeda, UCB, Ono, Integra Japan, IQVIA, Fuji Rebio, Biogen, and advisory fees from Janssen during the past three years. AP reports personal fees from Acadia Pharmaceuticals, Functional Neuromodulation, Neurim Pharmaceuticals, Grifols, Eisai, BioXcel, Tetra Discovery Partners, and Merck; grants from AstraZeneca, Avanir, Biogen, Biohaven, Eisai, Eli Lilly, Janssen, Genentech/Roche, Novartis, Merck, as well as funding from NIA, $\mathrm{NIMH}, \mathrm{DOD}$. KLP receives grant funding from the National Institute on Aging the National Institute of Neurological Disorders and Stroke, the Global Brain Health Institute, and Quest Diagnostics. BV has consultancy and research grants from Roche, Biogen, EISAI, Nestle, Lilly, Cerecin, and Merck. HH is an employee of Eisai Inc. and serves as Senior Associate Editor for the Journal Alzheimer's \& Dementia; during the past three years he had received lecture fees from Servier Biogen and Roche, research grants from Pfizer, Avid, and MSD Avenir (paid to the institution), travel funding from Eisai, Functional Neuromodulation, Axovant, Eli Lilly and company, Takeda and Zinfandel, GE-Healthcare and Oryzon Genomics, consultancy fees from Qynapse, Jung Diagnostics, Cytox Ltd., Axovant, Anavex, Takeda and Zinfandel, GE Healthcare, Oryzon Genomics, and Functional Neuromodulation, and participated in scientific advisory boards of Functional Neuromodulation, Axovant, Eisai, Eli Lilly and company, Cytox Ltd., GE Healthcare, Takeda and Zinfandel, Oryzon Genomics and Roche Diagnostics. $\mathrm{He}$ is co-inventor in the following patents as a scientific expert and has received no royalties: • In Vitro Multiparameter Determination Method for The Diagnosis and Early Diagnosis of Neurodegenerative Disorders Patent Number: 8916388; • In Vitro Procedure for Diagnosis and Early Diagnosis of Neurodegenerative Diseases Patent Number: 8298784; • Neurodegenerative Markers for Psychiatric Conditions Publication Number: 20120196300; • In Vitro Multiparameter Determination Method for The Diagnosis and Early Diagnosis of Neurodegenerative Disorders Publication Number: 20100062463; • In Vitro Method for The Diagnosis and Early Diagnosis of Neurodegenerative Disorders Publication Number: 20100035286 ; - In Vitro Procedure for Diagnosis and Early Diagnosis of Neurodegenerative Diseases Publication Number: 20090263822; • In Vitro Method for The Diagnosis of Neurodegenerative Diseases Patent Number: 7547553; • CSF Diagnostic in Vitro Method for Diagnosis of Dementias and Neuroinflammatory Diseases Publication Number: 20080206797; • In Vitro Method for The Diagnosis of Neurodegenerative Diseases Publication Number: 20080199966; • Neurodegenerative Markers for Psychiatric Conditions Publication Number: 20080131921. AV is an employee of Eisai Inc. and received lecture honoraria from Roche, MagQu LLC, and Servier.

Open Access: This article is distributed under the terms of the Creative Commons Attribution 4.0 International License (http:/ / creativecommons.org/ licenses/by/4.0/), which permits use, duplication, adaptation, distribution and reproduction in any medium or format, as long as you give appropriate credit to the original author(s) and the source, provide a link to the Creative Commons license and indicate if changes were made.

\section{References}

1. Jack CR, Bennett DA, Blennow K, et al. NIA-AA Research Framework: Toward a biological definition of Alzheimer's disease. Alzheimers Dement J
Alzheimers Assoc 2018;14:535-562

2. Petersen RC, Caracciolo B, Brayne C, Gauthier S, Jelic V, Fratiglioni L. Mild cognitive impairment: a concept in evolution. J Intern Med 2014;275:214-228

3. Bernstein A, Rogers KM, Possin KL, et al. Primary Care Provider Attitudes and Practices Evaluating and Managing Patients with Neurocognitive Disorders. J Gen Intern Med 2019;34:1691-1692

4. Bradford A, Kunik ME, Schulz P, Williams SP, Singh H. Missed and delayed diagnosis of dementia in primary care: prevalence and contributing factors. Alzheimer Dis Assoc Disord 2009;23:306-314

5. Olazarán J, Torrero P, Cruz I, et al. Mild cognitive impairment and dementia in primary care: the value of medical history. Fam Pract 2011;28:385-392

6. Morley JE, Morris JC, Berg-Weger M, et al. Brain health: the importance of recognizing cognitive impairment: an IAGG consensus conference. J Am Med Dir Assoc 2015;16:731-739

7. Hammers D, Spurgeon E, Ryan K, et al. Validity of a brief computerized cognitive screening test in dementia. J Geriatr Psychiatry Neurol 2012;25:89-99

8. Clionsky MI, Clionsky E. Development and validation of the Memory Orientation Screening Test (MOSTTM): A better screening test for dementia. Am J Alzheimers Dis Other Demen 2010;25:650-656

9. Ahmed S, de Jager C, Wilcock G. A comparison of screening tools for the assessment of mild cognitive impairment: preliminary findings. Neurocase 2012;18:336-351

10. Freedman M, Leach L, Carmela Tartaglia M, et al. The Toronto Cognitive Assessment (TorCA): normative data and validation to detect amnestic mild cognitive impairment. Alzheimers Res Ther 2018;10:65

11. Pfeffer RI, Kurosaki TT, Harrah CH, Chance JM, Filos S. Measurement of functional activities in older adults in the community. J Gerontol 1982;37:323329

12. Galvin JE, Roe CM, Powlishta KK, et al. The AD8: a brief informant interview to detect dementia. Neurology 2005;65:559-564

13. Jorm AF, Jacomb PA. The Informant Questionnaire on Cognitive Decline in the Elderly (IQCODE): socio-demographic correlates, reliability, validity and some norms. Psychol Med 1989;19:1015-1022

14. Chehrehnegar N, Nejati V, Shati M, et al. Behavioral and cognitive markers of mild cognitive impairment: diagnostic value of saccadic eye movements and Simon task. Aging Clin Exp Res 2019

15. Lin Y, Shan P-Y, Jiang W-J, Sheng C, Ma L. Subjective cognitive decline: preclinical manifestation of Alzheimer's disease. Neurol Sci Off J Ital Neurol Soc Ital Soc Clin Neurophysiol 2019;40:41-49

16. Rabin LA, Smart CM, Amariglio RE. Subjective Cognitive Decline in Preclinical Alzheimer's Disease. Annu Rev Clin Psychol 2017;13:369-396

17. CogniSenseTM iPad App for Cognitive Impairment Screening. http://www. questcognisense.com/. Accessed 14 Aug 2019

18. Possin KL, Moskowitz T, Erlhoff SJ, et al. The Brain Health Assessment for Detecting and Diagnosing Neurocognitive Disorders. J Am Geriatr Soc 2018;66:150-156

19. Buckley RF, Maruff P, Ames D, et al. Subjective memory decline predicts greater rates of clinical progression in preclinical Alzheimer's disease. Alzheimers Dement J Alzheimers Assoc 2016;12:796-804

20. van Harten AC, Mielke MM, Swenson-Dravis DM, et al. Subjective cognitive decline and risk of MCI: The Mayo Clinic Study of Aging. Neurology 2018;91:e300-e312

21. Hampel H, O'Bryant SE, Molinuevo JL, et al. Blood-based biomarkers for Alzheimer disease: mapping the road to the clinic. Nat Rev Neurol 2018;14:639-652

22. Henriksen K, O'Bryant SE, Hampel H, et al. The future of blood-based biomarkers for Alzheimer's disease. Alzheimers Dement J Alzheimers Assoc 2014;10:115-131

23. Lewczuk P, Ermann N, Andreasson U, et al. Plasma neurofilament light as a potential biomarker of neurodegeneration in Alzheimer's disease. Alzheimers Res Ther 2018;10:71

24. Janelidze S, Stomrud E, Palmqvist $S$, et al. Plasma $\beta$-amyloid in Alzheimer's disease and vascular disease. Sci Rep 2016;6:26801

25. Shi Y, Lu X, Zhang L, et al. Potential Value of Plasma Amyloid- $\beta$, Total Tau, and Neurofilament Light for Identification of Early Alzheimer's Disease. ACS Chem Neurosci 2019;10:3479-3485

26. Lue L-F, Sabbagh MN, Chiu M-J, et al. Plasma Levels of A $\beta 42$ and Tau Identified Probable Alzheimer's Dementia: Findings in Two Cohorts. Front Aging Neurosci 2017;9

27. Nakamura A, Kaneko N, Villemagne VL, et al. High performance plasma amyloid- $\beta$ biomarkers for Alzheimer's disease. Nature 2018;554:249-254

28. Xu H, Xiao S. Detection of plasma biomarker candidates for Alzheimer's disease and amnestic mild cognitive impairment. Alzheimers Dement J Alzheimers Assoc 2018;14:P1173-P1174 